\title{
Cyberpsychology: Video games as a perspective for cognitive training
}

\author{
Sana Raouafi ${ }^{*}$ and Faustin Armel Etindele Sosso ${ }^{2,3 *}$ \\ ${ }^{1}$ Biomedical Engineering Department, Polytechnic School of Montreal, Quebec, Canada \\ ${ }^{2}$ Research Center in Neuropsychology and Cognition, University of Montreal, Quebec, Canada \\ ${ }^{3}$ Department of Biological Sciences, Faculty of Arts and Sciences, University of Montreal, Quebec, Canada
}

\begin{abstract}
The current advances in preventive research on neurodegenerative diseases affecting memory suggests greater efficacy of treatments; In the face of cognitive deficits and associated early symptoms such as memory loss and dementia of the Alzheimer or Parkinson type, or medium- and long-term memory impairment caused by genetic or environmental disorders. As a result, early identification of patients at risk and better approaches to preserve their cognitive performance is a major global health issue. Cerebral training through cognitive IQ tests and video games with a more or less realistic personalized simulation environment; Has become a promising avenue that neuroscientists have been exploring for some years. This brief communication is a mini review simplified of the recent scientific advances, on the surprising effects on the brain of a cognitive training by means of interactive simulations or video games.
\end{abstract}

\section{Introduction}

The current advances in preventive research on neurodegenerative diseases affecting memory suggests greater efficacy of treatments; In the face of cognitive deficits and associated early symptoms such as memory loss and dementia of the Alzheimer or Parkinson type, or medium - and long-term memory impairment caused by genetic or environmental disorders. As a result, early identification of patients at risk and better approaches to preserve their cognitive performance is a major global health issue. Cerebral training through cognitive IQ tests and video games with a more or less realistic personalized simulation environment; Has become a promising avenue that neuroscientists have been exploring for some years. This brief communication is a mini review simplified of the recent scientific advances, on the surprising effects on the brain of a cognitive training by means of interactive simulations or video games.

\section{Action video games are the most effective}

Scientists have long questioned the best cognitive test, which can best adapt to the different clientele encountered in consultation, education or clinics. The answer to this question is reported in some recent publications that highlight the use by mental health professionals or cognitive disorders of various techniques to assess memory disorders already diagnosed in patients, or improve and slow down. The development or onset of these symptoms in healthy individuals. These tools range from cognitive assessment tests such as the McNair score, or multitasking tests performed under MRI. Regardless of the category of tools used, the key is to be able to effectively detect, evaluate and monitor the monitoring of the cognitive performance of our patients [1-4]. Video games prove to be more and more adapted for this brain training, despite their lack of evaluative specificity for any cerebral parameter. Contrary to what one could imagine, games of reflection such as the game of the lady, the game of chess or the tests of logic; Are not the ones that stimulate our brain the most. In fact, according to recent studies including participants of ages ranging from 8 to 65 years and various disciplines ranging from psychology to neurology; Action games are the most beneficial for the brain, whether online, on the phone or on the console. The visual environment is enriched with special effects and kinematics continuously stimulating the visual system and attention, forcing the subject to make quick and precise decisions. Modern action video games (those published since the mid-2000s, such as CALL OF DUTY, METAL GEAR SOLID and FIFA) have evolved following the performance of consoles and computers and require active learning (decision-making for example) [5-9]. This is more effective than passive learning, for the maintenance and development of cognitive functions $[10,11]$. Scientific studies highlight the much more lasting and significant positive effects: playing action video games would increase the ability to make quick decisions and ignore distractions, in that would even improve the creativity of children. Despite popular belief, it is now demonstrated that the effect of video games violent or not, is extremely weak on the behaviour of regular players; even if the latter show an aggressive immediately after playing $[12,13]$.

\section{Intensive practice maintains a healthy cognitive function}

According to a recent study, $73 \%$ of Americans aged 8-29 in North America play an average of 4 hours per week through their smartphones or computers. Players trained in action-based video

Correspondence to: Faustin Armel Etindele Sosso, Research Center in Neuropsychology and Cognition, University of Montreal, Quebec, Canada; Tel: +15143436111 \#3187; E-mail: Etindele@hotmail.fr

Key words: cyberpsychology, brain, neurodegenerative diseases, video games, training, cognitive impairment

Received: May 31, 2017; Accepted: June 16, 2017; Published: June 19, 2017 
games more easily follow a target in a complex environment, and their ability to concentrate increases considerably depending on the type of game. They develop a greater capacity for attention: A target or solve a puzzle in English while reading a subtitle in another language; And are less distracted by other objects near themselves with sound stimulation. They react faster, and they are also quicker to refocus their attention to the search for a new target in the virtual environment and develop reflexes above the average of the people. This has recently been associated with a significant increase in cognitive capacity for attention and memory, with a correlation between regular gaming practice and a change in the functioning of the hippocampus $[6,10]$. Those who play action games develop their vision, apprehending a greater number of objects at a glance. Their visual attention is better distributed in space [9]. They also get better results in acuity tests. These beneficial results are more interesting as video games are more geographically and financially accessible than a family physician in countries like Canada. They would be a possible preventive tool against visual and memory disorders with a possible reduction of consultations related to these disorders, if a regulation made some screening or prevention tools [14,15].

\section{Multi-sensory rehabilitation perspective}

There is an interesting potential for re-education in video games. Their educational potential also seems under-exploited. Introducing more complex, more exciting, more structured scenarios should increase their brain impact. Video games can potentially be used as therapy for patients with mental disorders causing a reduction or alteration of certain parts of the brain. The virtual environments of video games force the player to simultaneously use almost all cognitive functions. This leads to an increase in information processing capacities in the youngest (between 8 and 30 years), and a slowing of the cognitive decline [11,14,16-18]. These disorders include schizophrenia, post-traumatic stress disorder and Alzheimer's. Studies clearly show the rehabilitation of working memory, an increase in overall executive memory, as well as learning in people initially affected by an increase in gray matter in the brain [19]. The research also looks at the applications of video games in the correction of pathologies associated with the nervous systems. Several rapidly developing neuromuscular pathologies could benefit. For example, ataxia, which is a neuromuscular disorder characterized by a lack of coordination of the voluntary movements, is not caused by a muscular deficit but rather by an attack of the nervous system. This coordination disorder is partially corrected by visual control, and as neuroplasticity is greatly influenced by surrounding visual stimuli; It is therefore possible to improve coordination and treatment of visual and affective information in patients with advanced ataxia $[7,20,21]$. In conclusion, well outside of its distractive aspect and its recreational reputation; The practice of video games proves to be a promising therapy for cognitive pathologies. One can envisage a control of the symptomatic evolution of the cognitive disorders, as well as an early or palliative treatment of the associated pathologies already diagnosed.

\section{Conclusion}

The addiction for video games and entertainment should serves cognition and Cyberpsychology. It is possible to adjust the basic practice of entertainment assigned to video games, and to turn it in a supplementary tool for cognitive training. The usage above are a just a part of applications and interesting assignment of video games. Many projects currently ongoing in engineering and biomedicine, will certainly exhibits a lot of other benefits.

\section{Conflict of interests:}

Each the authors mentioned above, has no conflicts of interests, financial or otherwise with the present study

\section{Author contributorship:}

Faustin Armel Etindele Sosso and Sana raouafi contributed equally in the present research. Both work for conception and design of the study, experiments, analysis, and writing of the final version of manuscript.

\section{References}

1. Etindele Sosso F (2017) Sleep Disorders and Insomnia: Effects on a Young Population. Psychology and Psychiatry 2: 26-32.

2. Sosso FAE (2016) Neurocognitive game between risk factors, sleep and suicidal behaviour. Sleep Science.

3. Etindele Sosso FA, Raouafi S (2016) Brain Disorders: Correlation between Cognitive Impairment and Complex Combination. Mental Health in Family Medicine 12: 215-22.

4. Etindele Sosso F, Raouafi S (2016) Appropriate Sleep Duration and Physical Activity Modulate Cognitive Improvement. J Sleep Disor: Treat Care 5: 4.

5. Aliyari H, Kazemi M, Tekieh E, Salehi M, Sahraei H, et al. (2015) The Effects of Fifa 2015 Computer Games on Changes in Cognitive, Hormonal and Brain Waves Functions of Young Men Volunteers. Basic Clin Neurosci 6: 193-201. [Crossref]

6. Anguera JA, Boccanfuso J, Rintoul JL, Al-Hashimi O, Faraji F, Janowich J, et al (2013) Video game training enhances cognitive control in older adults. Nature 501: 97-101. [Crossref]

7. Bailey K, West R (2013) The effects of an action video game on visual and affective information processing. Brain Res 1504: 35-46. [Crossref]

8. Bavelier D, Davidson RJ (2013) Brain training : Games to do you good. Nature 494 425-426. [crossref]

9. Blacker KJ, Curby KM, Klobusicky E, Chein JM (2014) Effects of action video game training on visual working memory. J Exp Psychol Hum Percept Perform 40: 1992 2004. [Crossref]

10. Cardoso-Leite P, Bavelier D (2014) Video game play, attention, and learning: how to shape the development of attention and influence learning? Curr Opin Neurol 27: 185-91. [Crossref]

11. Clemenson GD, Stark CE (2015) Virtual Environmental Enrichment through Video Games Improves Hippocampal-Associated Memory. J Neurosci 35: 16116-25. [Crossref]

12. Chang CH, Pan WW, Chen FC, Stoffregen TA (2013) Console video games, postura activity, and motion sickness during passive restraint. Exp Brain Res 229: 235-42. [Crossref]

13. Zvyagintsev M, Klasen M, Weber R, Sarkheil P, Esposito F, et al. (2016) Violence-related content in video game may lead to functional connectivity changes in brain networks as revealed by fMRI-ICA in young men. Neuroscience 320: 247-58. [Crossref]

14. Anderson K, Grossberg GT (2014) Brain games to slow cognitive decline in Alzheimer's disease. J Am Med Dir Assoc 15: 536-7. [Crossref]

15. Deveau J, Jaeggi SM, Zordan V, Phung C, Seitz AR (2014) How to build better memory training games. Front Syst Neurosci 8: 243. [Crossref]

16. Boot WR, Champion M, Blakely DP, Wright T, Souders DJ, et al. (2013) Video games as a means to reduce age-related cognitive decline: attitudes, compliance, and effectiveness. Front Psychol 4: 31. [Crossref]

17. Foster PP (2015) Role of physical and mental training in brain network configuration. Front Aging Neurosci 7: 117. [Crossref]

18. Kim D, Kim HJ, Sasaki Y, Watanabe T, Pietrzak E, et al. (2014) Using Virtual Reality and Videogames for Traumatic Brain Injury Rehabilitation: A Structured Literature Review. Games Health J 3: 202-14. [Crossref]

19. Gong D, He H, Liu D, Ma W, Dong L, et al. (2015) Enhanced functional connectivity and increased gray matter volume of insula related to action video game playing. Sci Rep 5: 9763. [Crossref]

20. Shams TA, Foussias G, Zawadzki JA, Marshe VS, Siddiqui I, et al. (2015) The Effects of Video Games on Cognition and Brain Structure: Potential Implications for Neuropsychiatric Disorders. Curr Psychiatry Rep 17: 71. [Crossref]

21. Szycik GR, Mohammadi B, Hake M, Kneer J, Samii A, et al. (2016) Excessive users of violent video games do not show emotional desensitization: an fMRI study. Brain Imaging Behav [Crossref]

Copyright: (C2017 Raouafi S and Etindele Sosso FA. This is an open-access article distributed under the terms of the Creative Commons Attribution License, which permits unrestricted use, distribution, and reproduction in any medium, provided the original author and source are credited. 